\title{
La convergencia entre el conocimiento, las esferas estatales y las profesiones en la obra de Norbert Elías
}

\author{
The convergence between knowledge, state spheres, and \\ professions in Norbert Elias's work
}

María Eugenia De Ponti medeponti@gmail.com

http://orcid.org/0000-0002-7376-5472

Facultad Latinoamericana de Ciencias Sociales/

Universidad Nacional de Lanús (Argentina)

\section{Resumen}

El presente artículo intentará reconstruir una línea teórica desarrollada por Norbert Elías de manera parcial en diferentes textos de su obra. El objetivo principal es demostrar las incidencias mutuas existentes entre tres procesos: la complejización del conocimiento, la diferenciación de las esferas estatales y el surgimiento de nuevas profesiones. Se tomarán a los políticos profesionales y a los cientistas sociales como ejemplos de nuevas profesiones surgidas en el marco de un desarrollo social secularizado, caracterizado por un nivel elevado de interdependencia de las esferas sociales. Se buscará respetar la estructura conceptual esencial del autor y observar cómo sus conceptos principales -tales como desarrollo, psicogénesis, sociogénesis, figuración, entre otros- se vuelven fundamentales para comprender el proceso social que aquí se intentará reconstruir. 
Palabras clave: Conocimiento; interdependencia; esferas estatales; profesiones; poder.

\section{Abstract}

This article will try to reconstruct a theoretical line developed by Norbert Elías in various parts of his work. The main purpose is to show the mutual influences between three processes: the increasing complex of knowledge, the differentiation of state spheres and the arise of new professions. Professional politicians and social scientists will be used as examples of new professions grown in a secular society characterized by high levels of interdependence between different social spheres. This paper will use the author's conceptual structure to illustrate how concepts such as development, psychogenesis, sociogenesis, figuration, among others, are key to understand the convergence of the social processes already mentioned.

Keywords: Knowledge; interdependence; state spheres; professions; power.

Este trabajo buscará desarrollar la idea rastreada a lo largo de la obra de Elías sobre el modo en que el desarrollo del conocimiento actúa como una variable de peso a la hora de pensar el surgimiento de nuevas actividades profesionales. Este proceso se hace visible en el marco de la complejización creciente de las sociedades y de la también creciente interdependencia de los sujetos y de las esferas sociales. En este caso el foco estará puesto sobre dos profesiones en particular: los políticos profesionales y los científicos. A lo dicho anteriormente, se sumará el proceso de la evolución creciente de las estructuras estatales. Desde la teoría eliasiana, estos tres procesos -el desarrollo del conocimiento, el surgimiento de nuevas actividades profesionales y la evolución de las estructuras estatales-, se consideran como procesos dinámicos totalmente integrados e inseparables unos de otros.

De manera inicial, se desea esclarecer algunos conceptos que resultan esenciales tanto para la obra de Elías como para el objeto aquí planteado. En todos los desarrollos teóricos, el autor hizo hincapié en la falta de precisión con la que cuenta el lenguaje, así, por ejemplo, el concepto de desarrollo debería ser considerado como un concepto de función y no de acción, en pos de poder conceptualizar una sucesión impersonal y autorregulada de sucesos orientados en una determinada dirección (Elías, 2008) buscando desprenderse de la perspectiva economicista que propone una lógica de causa-efecto en la que el desarrollo es guiado por la acción de individuos puntuales. Es así que el autor considera a algunos 
conceptos tales como estructura y cambio, un tanto estáticos ya que relegan el cambio a momentos y a personas específicos, y no permiten evidenciar la dinámica inmanente a todo proceso social. Es en este sentido que en este trabajo se propone abordar la interrelación planteada desde esta perspectiva de desarrollo.

Adicionalmente, otros dos conceptos que actúan de base para la estructuración de este trabajo son el de interdependencia o dependencia mutua, y el concepto de figuración, ambos sumamente vinculados al concepto de desarrollo recién mencionado. Como se expresa en esa cita, "el concepto de figuración (sitúa)al problema de las interrelaciones humanas en el centro del planteamiento (...) de qué modo varían las interdependencias de los hombres al compás de la creciente diferenciación social y de la multiplicidad, siempre en aumento, de los planos de las sociedades" (Elías, 2008: 159). Lo anterior es indicativo de la concepción eliasiana sobre la formación de un sistema de interdependencias recíprocas que vuelve a los seres humanos cada vez más dependientes unos de otros, así como de las instituciones sociales. Como se ejemplifica para el caso de la realeza, el

(...) desarrollo personal del rey y el de su posición, van de la mano. Puesto que ésta posee una elasticidad específica, puede hasta cierto punto ser orientada según el desarrollo personal del que la ocupa. Pero cada posición social, aun la del rey absoluto, en virtud de su interdependencia respecto de otras posiciones del global sistema social al que pertenece, combina con su elasticidad una fuerza propia extraordinariamente grande, si se la compara con la de su detentor individual. La estructura de su posición ha fijado a su campo de acción estrictos límites (...) (Elías, 1996: 33).

Finalmente, los conceptos de psicogénesis y sociogénesis servirán de basamento para entender que los procesos sociales se pueden explicar bajo la lógica de una génesis tanto psicológica como social, indivisibles entre sí, lo que permite comprender el modo en que se anclan los procesos en los seres humanos, así como en la sociedad y viceversa. Tal como expresan Antón y Damiano, "No se trata de observar acciones o comportamientos aislados sino de mirarlos como configuraciones de acciones que expresan y estructuran relaciones sociales entre individuos, y que tienen una historia de desenvolvimiento: una psicogénesis y una sociogénesis" (2014: 6).

En base a estos conceptos mencionados, se buscará realizar inicialmente una síntesis sobre el desarrollo del conocimiento a nivel general, y del conocimiento secular en particular, retomando parte de la sociología del conocimiento del autor. Luego se intentará abordar el desarrollo de nuevas prácticas profesionales basadas en esferas del conocimiento altamente especializadas 
enmarcadas en un desarrollo de las instituciones del Estado también altamente diferenciadas. A lo largo del trabajo, se hará presente el poder como una dimensión que es parte constitutiva de las relaciones de los individuos y que influye en el proceso social que se intenta abordar.

\section{Desarrollo del conocimiento}

Según el autor, "(...) lo que llamamos conocimiento es el significado social de símbolos construidos por los hombres tales como palabras o figuras, dotados con capacidad para proporcionar a los humanos medios de orientación" (Elías, 1994a: 55). A esta conceptualización general sobre el conocimiento, se suma la característica esencial del ser humano que le permite adquirir estos símbolos -y sus significados- por medio del aprendizaje y así guiarse por ellos. Este proceso, denominado por Elías (1994) comoemancipación simbólica, se complementa con el hecho de que los símbolos son "intercambiables", es decir que pueden ir mudando conforme se vayan complejizando o bien modificando su simbolización. Es en función de esta concepción del desarrollo del conocimiento que el autor afirma que el lenguaje actúa como un modo de regularización social (Elías, 1994a).

A lo largo de la historia, los medios de orientación actuaron como guías para la acción, desde funciones básicas como la supervivencia y las relaciones interpersonales de socialización primaria en sociedades poco diferencias con escasos planos de integración e interdependencia, hasta las sociedades actuales caracterizadas por altos niveles de diferenciación, y por numerosos planos de integración altamente interdependientes. En este marco, no es posible hablar de conocimiento y de medios de orientación sin hacer referencia a la dimensión del poder (1), ya que Elías considera que la posibilidad y/o la capacidad de un individuo o de un grupo de individuos de controlar tanto las pautas de conocimiento como los medios de orientación -considerados aquí como una de las posibles fuentes de poder-, es correlativa con la capacidad de un tal grupo de imponerse sobre otro, lo que genera un desequilibrio de poder (Elías, 1994b: 39).

Bajo un escenario del conocimiento como fuente de poder, pueden rastrearse por lo menos tres etapas históricas que expresan de manera clara el lugar que ocupa el conocimiento en el proceso civilizatorio. Históricamente, la iglesia católica fue la gran monopolizadora del conocimiento tanto porque eran los únicos que manejaban la lectura y la escritura, como porque se erigieron como los "elegidos" para la revelación del conocimiento mágico-mítico. Esto los colocó hasta el Renacimiento como los grandes monopolizadores de los medios de orientación. La relación entre los sacerdotes y losguerreros nobles -encargados del monopolio 
de la violencia física- se reflejaba en un equilibrio de poder que comenzó a cambiar cuando los segundosiniciaron una avanzada sobre otras funcionesque desempeñaban los sacerdotes, generando un desequilibrio en la balanza de poder que, hasta ese momento, había mantenido a la iglesia católica en la cima. Tales funciones tiene que ver con ocupar espacios relativos a las instituciones de gobierno existentes en aquel entonces, así como también con el control de los medios de producción.

Alrededor del siglo XV los guerreros -como representantes de los príncipes y de la noblezainvirtieron la relación, y pasaron a ocupar los espacios de poder más importantes. En esta misma transición, se produjo el desbloqueo del monopolio de los medios de orientación y del conocimiento, ya que paulatinamente comenzó a darse un proceso de desarrollo secular que invalidó a la revelación mágico-mítica como el único modo de acceso al conocimiento, y como correlato, se incluyó a la comprobación empiríca como medio de refutación del conocimiento que se iba construyendo. El hecho de que el conocimiento secular sea corroborable, lo vuelve en contra de la autoridad establecida (Elías, 1994a: 76).

De esta manera, la importancia de este conocimiento, no es sólo su capacidad de ser comprobado empíricamente, sino que también incluyó tendencias fuertemente antiautoritarias. Lo que el autor denomínó democratización funcional (Elías, 1994a) del conocimiento, tiene quever con el creciente poder de la población por sobre los gobernantes, que podría vehiculizarse por medio de una reciprocidad institucional de control, y no bajo una única vía de gobernantes hacia gobernados (2).

Lo descripto anteriormente permite observar un indicador del grado de desarrollo y de diferenciación social (3) ya que un único grupo social podía ser responsable por más de una función social básica y satisfacer esencialmente todas las demandas sociales -lo que le permite ubicarse en una posición de poder-. Elías (1998) menciona algunas funciones básicas indispensables para el desarrollo de las sociedades. Algunos ejemplos que él considera que formaron parteimprescindible del desarrollo social -pero pueden aparecer otros- son la monopolización de la producción alimenticia, del control de la violencia, del conocimiento, de los tributos y de la presión para el ejercicio de la autocoacción.

Sólo para mencionar otros ejemplos, el monopolio del conocimiento y de los medios de orientación también jugaron un papel importante en la Rusia soviética (Elías, 1998: 259). En la actualidad, no se presenta un caso de monopolio, pero sí de una importancia fundamental del conocimiento y de un tipo de conocimiento particular -el científico- que ha alcanzado altos niveles de especialización. En ambos casos se puede observar cómo el conocimiento actúa como una fuente de poder. 


\section{Desarrollo de las esferas estatales y surgimiento de nuevas profesiones}

El análisis de los procesos sociales en el largo plazo es una de las batallas teórico metodológicas que Elías buscó defender a lo largo de toda su obra. Es desde esta perspectiva que afirma que“Un análisis sociológico-evolutivo más preciso muestra claramente que la evolución de las estructuras estatales y profesionales son dos aspectos indisolubles del desarrollo de un solo marco de funcionamiento social general" (Elías, 2008: 168). Este marco de funcionamiento social general incluye una fuerte eclosión del conocimiento que le permitió a la sociedad ir aumentando sus planos de diferenciación y en consecuencia, el nivel de interdependencia entre las personas. Uno de los pasos cruciales para alcanzar altos niveles de interdependencia y para la conformación de ese marco de funcionamiento social general, fue lo que el autor llamó un proceso bifurcado de secularización (Elías, 1994), haciendo referencia al surgimiento de un nuevo tipo de conocimiento, el conocimiento secular, como ya fuera dicho más arriba, así como a un proceso de surgimiento y fortalecimiento de gobiernos de Estado.

No obstante el hecho de que tales procesos ya se estaban sucediendo, el autor resalta dos ejemplos vinculados por un lado, con la poca distinción que se evidenciaba entre las esferas públicas y privadas de la vida de los guerreros nobles, y por otro lado, con la ocupación de cargos de poder en diferentes tipos de acitvidades. En este sentido,

(...) en los Estados Nacionales industrializados, los organismos de control público -el parlamento, la prensa, la judicatura o los partidos que competían abiertamente por el poderobligaron a distinguir con relativa claridad los asuntos personales y los oficiales, aun en el caso de los hombres y mujeres más poderosos del Estado (Elías, 1996: 9).

Esto permite evindenciar el modo en que los procesos cuentan con un doble anclaje integrado desde el cual se pueden comprender: la psicogénesis observada en la conducta de los individuos, así como la sociogénesis en los procesos generales de desarrollo social.

En El Proceso de la Civilización (1987) el autor describe claramente el modo en que se conjugan las diferentes esferas sociales y el desarrollo de las capacidades estatales:

(...) el mismo florecimiento de la división del trabajo, del aseguramiento de los caminos y de los mercados en grandes superficies territoriales, la acuñación de moneda y del conjunto del gráfico monetario, la protección de la producción pacífica frente a la violencia física y otras medidas de coordinación y de regulación dependen en gran parte de la constitución 
de grandes centros de poder monopolista y centralizado. En otros términos, cuanto más se diferencien en una sociedad los procesos laborales y el conjunto de funciones, cuanto más largas y complicadas sean las cadenas de las acciones individiduales que están en interrelación con el fin de que las acciones individuales alcancen sus objetivos sociales, tanto más claramente aparece un rasgo distintivo del órgano central: el carácter del órgano supremo de coordinación y regulación para el conjunto del proceso de división de funciones (Elías; 1987: 394).

Ese órgano supremo mencionado por el autor, no es otro que el Estado.

Al interior de este proceso, se va dando el surgimiento de grupos especializados en conocimientos específicos capaces de desempeñar actividades puntuales, desapareciendo así la situación que se daba con los sacerdotes y los guerreros nobles, que se hacían cargo de varias funciones vitales al mismo tiempo. Uno de los nuevos grupos profesionales que es retomado por Elías es el de los políticos profesionales (1994a: 213), surgidos en el marco de los ya existentes partidos de masas.

Los políticos profesionales sintetizan también la representación de las esferas económicas -al igual que se venía dando con otros tipos de representantes- pero desde un conocimiento más diferenciado que incluye tanto la incorporación de otras variables en la representación, así como también, y particularmente, del know how de la acción polítca en las esferas estatales de gobierno.

Elías afirma que los políticos profesionalescomponen una nueva formación social que frecuentemente sobrepasa el poder de los grupos económicos (1994a: 213). No obstante en este trabajo seesboza la hipótesis -que será desarrollada en otro trabajo- de que históricamente se han percibido casos en que políticos profesionales y grupos económicos potencian su poder de manera interrelacionada, es decir que proponen un equilibrio de poder donde, los políticos que se vinculan con los empresarios, frecuentemente cuentan con mayor poder relativo que quienese se vinculan con las clases trabajadoras. Como afirma Elías, "Se podría decir que la proporción de poder de un grupo de funcionarios sociales varía de acuerdo con la cambiante fuerza e intensidad de las demandas sociales que dicho grupo puede satisfacer en virtud de su especialización" (1994a: 214), en este sentido, podría hipotetizarse que una de las demandas sociales capaces de satisfacer por parte de los políticos profesionales, es su reprensentación de los grupos económicos en las estructuras de gobierno. Históricamente los grupos empresarios han contado con mayor representación en tales estructuras en comparación con las clases trabajadoras. 
Retornando la relación existente entre el surgimiento de los políticos profesionales como correlato de la diferenciación creciente de las esferas estatales, se puede percibir también cómo tanto los políticos profesionales como los partidos de masas, se desarrollan de la mano de la especialización del conocimiento. Observar esta situación permite notar que, en la medida en que el desarrollo de la sociedad se presente como sumamente vinculado a las instituciones políticas, las posiciones de poder de éstas últimas así como de sus participantes aumentará de manera correlativa, "los partidos ponen de relieve claramente el grado de desigualdadque existe en el equilibrio de poder entre gobernantes y gobernados". (Elías, 1994: 61). Desde esta observación, el autor insiste en la necesidad de establecer instancias de control desde los gobernados a los gobernantes, es decir una reciprocidad institucional de control, como ya fuera mencionado más arriba.

Para esto, Elías considera necesario el desarrollo de conocimiento sociológico especializado que permitaentender de manera más comprehensiva los procesos sociales, con el objetivo de poder proveer a los hombres de medios de orientación y poder tener un mejor control de tales procesos, "cuanto más incontrolable sea para el hombre un contexto determinado, tanto más afectivo será su pensamiento acerca de él y cuanto más afectivo (...), tanto menos estára en condiciones de elaborar modelos adecuados" (Elías, 2008: 187) para su control.

Elías identifica varios problemas en relación a la posibilidad de construir ese concimiento especializado recién mencionado. Por un lado, considera que es necesario desarrollar una teoría del desarrollo de la ciencia que permita construir "(...) un fondo dado de conocimiento científico en tanto que sistema más o menos coherente y consistente" (Elías, 1994a: 169), aunque quienes históricamente han trabajado en ella, historiadores y filósofos, no cuenten aún con un modelo teórico que sea útil para decidir la selección y el ordenamiento de los desarrollos científicos en pos de la construcción de tal fondo de conocimiento. A diferencia de esto, tanto historiadores como filósofos suelen usar criterios indidviduales o relativos a la tradición científica a la cual pertenecen, por lo que resultaría poco fructífero combinar estudios que se realizaron bajo criterios teórico metodológicos parcial o totalmente arbitrarios (1994a: 176). Asimismo, Elías señala que no se realiza un correcta consideración de la comunidad científica como sujeto constructor del conocimiento con una evolución inmanente en constante cambio, así como tampoco de la autonomía relativa inherente al proceso de desarrollo científico, relacionada con la estructura social general, pero no signada unilaterlamente por ésta.

Por otro lado, las ciencias sociales aún atraviesan procesos primitivos en su desarrollo y se encuentran todavía muy influenciadas por distintos sistemas de creencias. A diferencia de 
(...) las investigaciones de las ciencias de la naturaleza, aunque no están, en absoluto, 'exentas de valores', el tipo de valoraciones que predomina en los trabajos de los científicos que estudian la naturaleza no se halla determinado por puntos de vista extracientíficos. En esto se diferencian, en el estado actual, las ciencias naturales de las sociales. En éstas últimas, la influencia de valoraciones externas, de tomas de partido en conlictos que afectan al conjunto de la sociedad, invaden la labor científica; la influencia de valoraciones heterónomas es, pues, muy grande (Elías,1990: 57).

En ese sentido, Elías considera que las ciencias sociales no son concientes de que aún queda un largo camino por recorrer en la lucha por una autonomía relativa y por una emancipación respecto a los ideales políticos y sociales de nuestros días.

Finalmente, el último problema que observa Elías en la comunidad académica tiene que ver con la comunidad científica en general, y con aquellos que se dedican exclusivamente a las Ciencias Sociales. El surgimiento de los científicos como una actividad profesional específica, guarda las mismas características que la aparición de políticos profesionales, es decir que también surgieron en un momento dado como parte del proceso de desarrollo social, del creciente conocimiento especializado y de la creciente interdependencia de las esferas de la sociedad. De igual manera, se asemeja a los políticos profesionales porque desarrollan una actividad que cuenta con características que les permitiría ubicarse en altas posiciones de poder. No obstante, Elías observa algunas diferencias en los modos de desarrollar ambas profesiones que influyen en la comunidad científica y en su ubicación en posiciones relativamente bajas en las relaciones de poder: un alto nivel de individualización; escasa organización colectiva; su distribución espacial en diferentes centros de estudio suele ser perjudicial para la construcción de una representación corporativa; a diferencia de los sacerdotes, como ya no pueden monopolizar el acceso al concocimiento, utilizan otros modos que dificultan el acceso, como por ejemplo un lenguaje demasiado tecnificado, generando dificultades para su difusión, entre otras (Elías, 1998: 288-289).

Estas distinciones que por ahora posee la comunidad científica deberían ser obejtivadas y tenidas en cuenta a la hora de construir ese acervo de conocimiento que el autor plantea, en pos de poder otorgar mejores medios de orientación a los individuos y mejores capacidades de control por sobre los gobernantes, por medio del conocimiento de las condiciones existentes.

\section{A modo de conclusión}


Este trabajo buscó desentrañar la relación existente entre el creciente desarrollo social y el surgimiento de nuevas esferas estatales, con el desarrollo del conocimiento especializado y con el nacimiento de nuevas actividades profesionales. El objetivo fue poder ofrecer algunas herramientas para entender cómo el conocimiento forma parte constitutiva del desarrollo social y en particular de la esfera politica y de la generación de nuevas profesiones, así como la importancia de no ceñir la consideración del conocimiento a un tipo de conocimiento únicamente científico, sino que para poder generar diagnósticos actuales es necesario incluir una teoría del conocimiento más amplia y a largo plazo.

De igual manera, se intentó respetar las perspectivas del autor en relación al largo plazo y a la consideración integral y multicausal del objeto de estudio. Así, se trató de dejar en claro que las partes de la relación aquí tratada no tienen una única causa, sino que forman parte de un entramado interdependiente con desarrollo relativamente autónomo, pasible de ser parcialmente conocido y parcialmente controlado pero que cuenta con una dinámica inherente a su propio desarrollo.

\section{Notas}

(1) Tal como afirma Marín (en Antón y Damiano, 2014): "Si comenzamos por conceptualizar al poder como una dimensión es para enfatizar el carácter multivariable del mismo, ya se considere su 'naturaleza', sus fuentes, su distribución o sus formas de manifestarse; y a su vez para poner de relieve su especificidad como 'proceso de construcción de relaciones sociales"'.

(2) Como mencionan Antón y Damiano los procesos de democratización funcional contribuyen a "la incorporación a la toma de decisiones de grupos antes excluidos de las mismas" (2014: 11).

(3) Para poder analizar el estado de desarrollo de una sociedad en el largo plazo, Elías (2008) propone una tríada de controles básicos: a. la dimensión de sus posibilidades de controlar el entorno extrahumano, es decir los hechos naturales; b. sus posibilidades de controlar las relaciones interhumanas, o sea las conexiones sociales; y c. por la dimensión del autocontrol.

\section{Bibliografía}

Anton, G. y Damiano, F. (2014). La dimensión poder en la obra de Norbert Elías. Prácticas de oficio. Investigación y reflexión en Ciencias Sociales, 14, 1-18.

Elías, N. (1990). Compromiso y distanciamiento. Barcelona: Península.

Elías, N. (1994a). Conocimiento y poder. Madrid: La Piqueta.

Elías, N. (1987). El proceso de la civilización. Madrid: Fondo de Cultura Económica. 
Elías, N. (1998). La civilización de los padres y otros ensayos. Bogotá: Norma.

Elías, N. (1996). La sociedad cortesana. México: Fondo de Cultura Económica.

Elías, N. (2008). Sociología Fundamental. Barcelona: Gedisa.

Elías, N. (1994b). Teoría del Símbolo. Un ensayo de antropología cultural. Barcelona: Península. 